\title{
In vivo Growth Kinetics of the Natural Course of an Undifferentiated Untreated Endometrial Cancer: An Observational Case Report
}

\begin{abstract}
We describe a patient with a histologically verified undifferentiated endometrial cancer who initially declined treatment, thus permitting study of the in vivo growth kinetics of the tumor. 266 days later, the patient wasdiagnosed with extensive symptomatic pelvic, abdominal, mediastinal, lung and supraclavicular metastases confirmed by imaging. During the untreated phase of 9.2 months, the tumor volume doubling time was estimated to be $56+3.5$ (SD; standard deviation) days when an exponential tumor growth was assumed. This rapid growth was paralleled by immunohistochemistry showing a higher mitotic rate in the metastasis than in the primary tumor. At a late stage of the disease, laparotomy showed pelvic extension of the endometrial tumor, parenchymal liver metastasis and peritoneal carcinomatosis. The patient died 1.5 years after the initial diagnosis. To the authors' knowledge, this is the first analysis of hypothetically generated exponential tumor kinetics of an untreated gynecologica cancer in vivo. This report may serve as a reference for assessing future clinical interventions in endometrial cancer.
\end{abstract}

Keywords: Endometrial cancer; Natural course of disease; In vivo Growth kinetics; Metastasis

\section{Introduction}

We describe a patient with an undifferentiated endometrial cancer who initially declined treatment, thus permitting study of the early hypothetical in vivo growth kinetics of the tumor and the natural history of the disease.

\section{Methods}

A 49-year-old nullipara presented with menometrorrhagia of two months' duration. Transvaginal sonography showed an inhomogeneous, 36-mm thick endometrium. Sonographically, deep myometrial invasion was suspected. FIGO stage Ia was diagnosed (tumor confined to the uterus and uterine sond length $<8 \mathrm{~cm}$; clinical FIGO staging system 1971). Endometrial aspiration biopsy revealed undifferentiated endometrioid adenocarcinoma. The patient denied magnetic resonance and computed tomography investigations. There was no clinical evidence of metastasis as established by physical examination, chest X-ray, and abdominal sonography. Initially the patient refused therapy due to personal reasons. Her sister had died from another malignancy a year ago. 9.2 months after diagnosis the patient returned with symptomatic pelvic, abdominal, mediastinal, lung and supraclavicular metastases confirmed by imaging (Table 1). Laparotomy showed pelvic extension of the endometrial tumor, parenchymal liver metastasis and peritoneal carcinomatosis. Cervical biopsy and omentectomy were performed but, due to extensive disease in the pelvis, the uterus was left in situ. Histology confirmed primary undifferentiated endometrial carcinoma. Subsequently, the

\section{Journal of} Andrology \&
Gynaecology

\author{
Edgar Petru*, Peter Regitnig ${ }^{3}$, Manuela Aschauer ${ }^{4}$ \\ and Haro Stettner ${ }^{2}$ \\ ${ }^{1}$ Departments of Obstetrics and Gynecology, Medical University of \\ Graz, Austria \\ ${ }^{2}$ Institute of Mathematics, Alpe Adria University of Klagenfurt, \\ Klagenfurt, Austria \\ ${ }^{3}$ Institute of Pathology, Medical University of Graz, Austria \\ ${ }^{4}$ Departments of Radiology, Medical University of Graz, Austria \\ Address for Correspondence \\ Edgar Petru, MD, Department of Obstetrics and Gynecology, Medical \\ University of Graz, Division of Gynecology, Auenbruggerplatz \\ 14, A-8036 Graz, Austria, Tel: +43.316.385.81082; Fax: \\ +43.316.385.12546; E-mail: edgar.petru@medunigraz.at \\ Submission: 15 August 2013 \\ Accepted: 17 September 2013 \\ Published: 20 September 2013
}

patient underwent palliative chemotherapy (starting on day 275 after initial diagnosis; Table 1). Metastases partially responded to chemotherapy but the patient died of bowel obstruction 1.5 years after initial diagnosis. Autopsy confirmed primary undifferentiated endometrial carcinoma.

\section{Calculating tumor growth kinetics}

At first presentation, the primary tumor was estimated to measure $36 \times 36 \mathrm{~mm}$ in diameter. The patient initially denied treatment and remained untreated until 266 days after diagnosis. At that time, the patient returned with signs of extensive tumor progression (Table 1). 24 lesions were diagnosed by CT of the chest and abdomen and MRI of the pelvis. All lesions were radiologically reviewed and systematically measured. The complete tumor volume was calculated as the sum of all lesions on day 266. The three largest lesions were measured in three dimensions. The remaining lesions were measured in 2 dimensions and the third dimension was estimated as the mean of the two other measured dimensions with $20 \%$ scattering.

Total tumor burden was estimated with the established standardized exponential model [1-3] using the formula:

Increase of tumor volume over time $(\mathrm{dV})$ from time $\mathrm{t}$ (day 0 ) to $\mathrm{dt}$ (day 266) in proportion $(=\lambda=$ growth parameter) to tumor volume at the time of diagnosis $=\mathrm{V}(\mathrm{t})$ :

$$
\mathrm{dV}=\lambda \times \mathrm{V}(\mathrm{t}) \times \mathrm{dt}
$$

\section{Immunohistochemistry}

Paraffin-embedded $4-\mu \mathrm{m}$ sections were stained and mitoses counted in 10 consecutive (HPF, X400). Immunohistochemistical staining for Ki-67, the cell-cycle dependent protein Cyclin D1, and the anti-apoptosis inhibitor $\mathrm{Bcl} 2$ was performed and the percentage of nuclei staining positive for MIB1, Cyclin D1 or Bcl2 was determined.

\section{Results}

Table 1 describes the clinical course of disease in the patient. Table 2 describes the changes of MIB1, Cyclin D1 and Bcl2 from the primary tumor at diagnosis to omental metastasis at late stage disease. 
Citation: Petru E, Regitnig P, Aschauer M, Stettner H. In vivo growth kinetics of the natural course of an undifferentiated untreated endometrial cancer: An observational case report. J Androl Gynaecol. 2013;1(1): 4.

Table 1: Clinical course of the 49-years old patient diagnosed with an undifferentiated endometrial cancer who initially refused therapy.

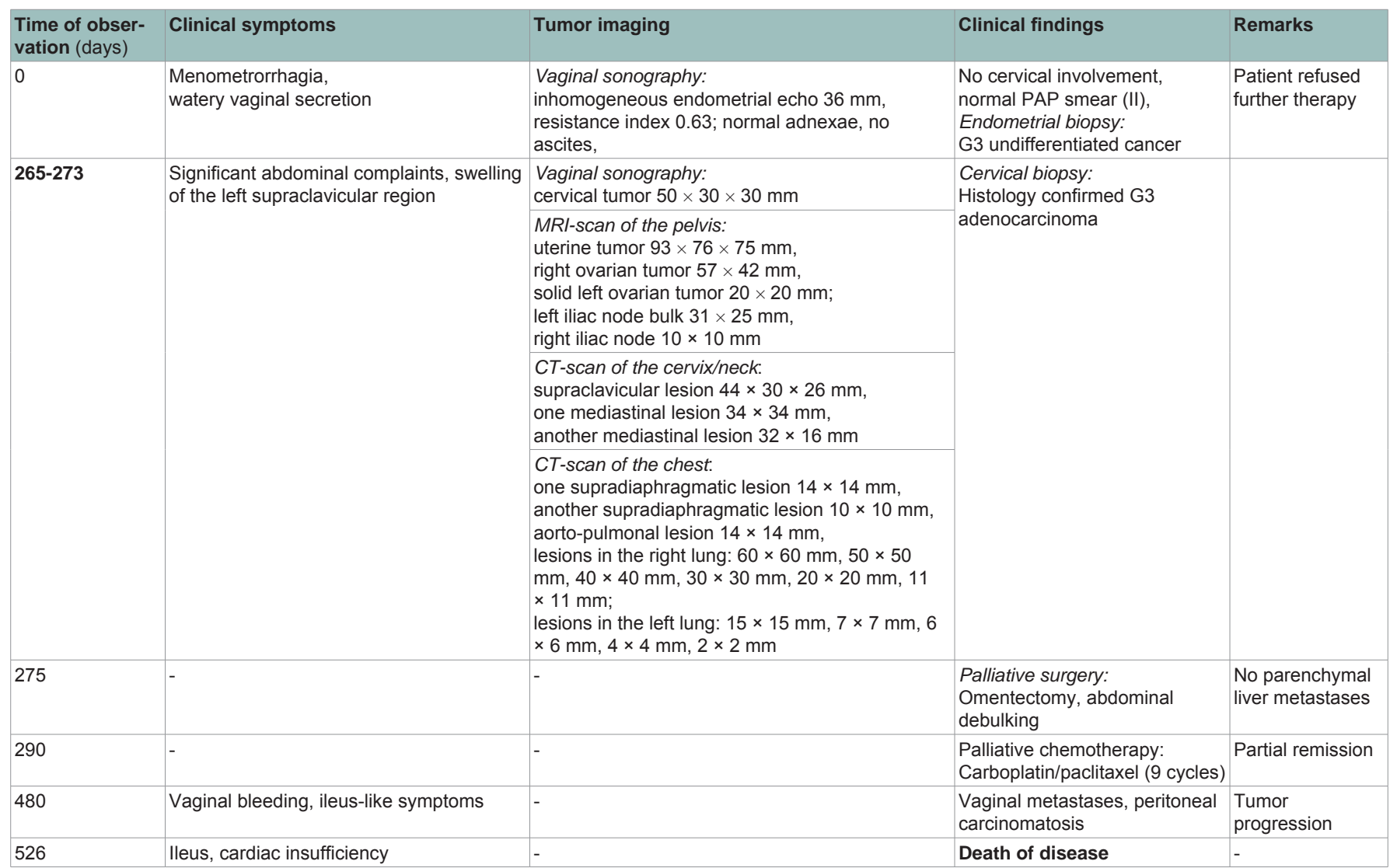

Table 2: Histopathologic and immunohistochemical characteristics of the primary endometrial tumor as opposed to its metastasis diagnosed 275 days later at laparotomy.

\begin{tabular}{|l|c|c|}
\hline & Primary endometrial tumor & Peritonealmetastasis \\
\hline Mitoses & $12 / 10 \mathrm{HPF}^{*}$ & $64 / 10 \mathrm{HPF}$ \\
\hline Mib1 & $31 \%$ & $48 \%$ \\
\hline Cyclin D1 & $84 \%$ & $86 \%$ \\
\hline Bcl-2 & $0 \%$ & $0 \%$ \\
\hline
\end{tabular}

Legend: * High-power fields

Tumor volume on day 0 was estimated to be $46.7 \mathrm{ccm}$ while it was $1270.8 \mathrm{ccm}$ on day 266 , respectively. The estimated tumor volume doubling-time was $56 \pm 3.5$ (standard deviation) days. Figure 1 shows the exponential curve of the hypothetical tumor growth from day 0 (initial diagnosis) to day 266, at which extensive metastases were diagnosed radiologically.

\section{Discussion}

In this patient with a primary undifferentiated endometrial cancer presented here, rapid tumor development over 9.2 months was demonstrated clinically and radiologically (Table 1). A hypothetical exponential tumor growth model was generated. On this basis, tumor volume doubling time was calculated to be 56 days (Figure 1). Rapid growth was paralleled by immunohistochemistry showing a higher mitotic rate in the metastasis than in the primary tumor (Table 2).

In endometrial cancer, it is not clear whether the duration of symptoms correlates with prognosis [4-6]. Little is known about growth rates of human gynecologic tumors in vivo. There exist only a few studies in which the natural growth of tumors has been determined. Among 70 patients diagnosed with meningeomas,
16 grew according to an exponential growth pattern [7]. Similar observations were found by Nakasu et al. [8] who described a quasiexponential growth patterns in six atypical menigeomas. Exponential tumor growth was also demonstrated in mouse liver tumors in vivo [9] and in tumor xenograft studies [10].

No accepted standard for describing in vivo growth kinetics exist in malignant (gynecologic) tumors. However, even the best growth model will unlikely reflect the growth situation of specific human malignant tumors in vivo. Heterogeneous tumor cell populations may exert various exponential growth rates [11]. The complicated human biological system also has to consider a variety of other influences including those of the immune system [12]. Furthermore, exponential malignant tumor growth in the early phase may be followed by a linear one [13].

Nevertheless, our observation of the natural course of an undifferentiated endometrial cancer gives a good opportunity to create hypotheses. One is that the tumor followed an exponential growth pattern although there is no prove for it. However, according to previous reports in vivo (7-10), exponential growth - as described for our patient - may have occurred and seems to be justified. 
Citation: Petru E, Regitnig P, Aschauer M, Stettner H. In vivo growth kinetics of the natural course of an undifferentiated untreated endometrial cancer: An observational case report. J Androl Gynaecol. 2013;1(1): 4.

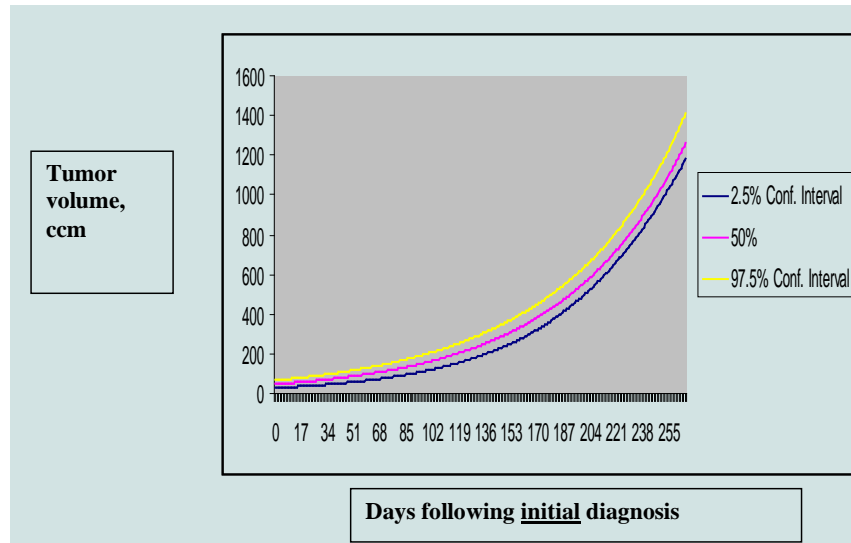

Figure 1: Estimated exponential tumor growth of the untreated endometrial cancer

To our knowledge, this is the first detailed report on hypothetic tumor kinetics of the natural course of an untreated gynecological cancer in vivo. It may serve as the reference for assessing future clinical interventions in endometrial cancer.

\section{References}

1. Armitage P, Colton T (2005) (Eds) Encyclopedia of biostatistics. (2 $2^{\text {nd }}$ edition), J. Wiley \& Sons, New York.

2. von Mangoldt H, Knopp K (1990) (Eds) Einführung in die höhere Mathematik (1 $7^{\text {th }}$ edition), Hirzel Verlag, Stuttgart.
3. Smirnow WI (2004) (Ed) Lehrgang der höheren Mathematik I. (16th edition ), Deutsch Verlag, Frankfurt/Main.

4. Johnson N, Miles T, Bailey D, Tylko-Hill K, Das N, et al. (2011) Delays in treating endometrial cancer in the south west of England. $\mathrm{Br} \mathrm{J}$ Cancer 104: 1836-1839.

5. Menczer J (2000) Diagnosis and treatment delay in gynecological malignancies. Does it affect outcome? Int J Gynecol Cancer 10: 89-94.

6. Smith EM, Anderson B (1987) Symptomatology, delay, and stage of disease in endometrial cancer. Cancer Detect Prev 10: 247-254.

7. Hashiba T, Hashimoto N, Izumoto S, Suzuki T, Kagawa N, et al. (2009) Serial volumetric assessment of the natural history and growth pattern of incidentally discovered meningiomas. J Neurosurg 110: 675-684.

8. Nakasu S, Nakasu Y, Fukami T, Jito J, Nozaki K (2011) Growth curve analysis of asymptomatic and symptomatic meningiomas. J Neurooncol 102: 303-310.

9. Schmid A, Rignall B, Pichler B, Schwarz M (2012) Quantitative analysis of the growth kinetics of chemically induced mouse liver tumors by magnetic resonance imaging. Toxicol Sci 126: 52-59.

10. Wu J (2011) Assessment of antitumor activity for tumor xenograft studies using exponential growth models. J Biopharm Stat 21: 472-483.

11. Mehara E, Forssell-Aronsson E, Johanson V, Kölby L, Hultborn R, et al. (2013) A new method to estimate parameters of the growth model for metastatic tumours. Theor Biol Med Model 10: 31.

12. Wen S, Ager E, Christophi C (2013) Bimodal role of Kupffer cells during colorectal cancer liver metastasis. Cancer Biol Ther 14: 606-613.

13. Rocchetti M, Germani M, Del Bene F, Poggesi I, Magni P, et al. (2013) Predictive pharmacokinetic-pharmacodynamic modeling of tumor growth after administration of an anti-angiogenic agent, bevacizumab, as singleagent and combination therapy in tumor xenografts. Cancer Chemother Pharmacol 71: 1147-1157. 